\title{
Social participation patterns and the incidence of functional disability: The Japan Gerontological Evaluation Study
}

Shigekazu Ukawa, Akiko Tamakoshi, Yutaka Okada, Yoichi M Ito, Rika Taniguchi, Yukako Tani, Yuri Sasaki, Junko Saito, Maho Haseda, Naoki Kondo, Katsunori Kondo

\begin{tabular}{|c|c|}
\hline Citation & Geriatrics and Gerontology International. 20(8); 765-772 \\
\hline Issue Date & $2020-06-30$ \\
\hline Type & Journal Article \\
\hline Textversion & author \\
\hline Rights & $\begin{array}{l}\text { This is the peer reviewed version of the following article: Geriatrics and Gerontology } \\
\text { International. Vol.20, Issu.8, p.765-772, which has been published in final form at } \\
\text { https://doi.org/10.1111/ggi.13966. This article may be used for non-commercial purposes } \\
\text { in accordance with Wiley Terms and Conditions for Use of Self-Archived Versions. }\end{array}$ \\
\hline DOI & 10.1111/ggi.13966 \\
\hline
\end{tabular}

Self-Archiving by Author(s)

Placed on: Osaka City University Repository

UKAWA S., et al. (2020). Social participation patterns and the incidence of functional disability: The Japan Gerontological Evaluation Study. Geriatrics and Gerontology International. 20, 765-772. Doi: 10.1111/ggi.13966 
Social participation patterns and the incidence of functional disability: The Japan

\section{Gerontological Evaluation Study}

Authors: Shigekazu Ukawa ${ }^{* 1,2}$, Akiko Tamakoshi ${ }^{2}$, Yutaka Okada ${ }^{3}$, Yoichi M. Ito ${ }^{4}$, Rika

Taniguchi $^{5}$, Yukako Tani ${ }^{6}$, Yuri Sasaki ${ }^{7}$, Junko Saito ${ }^{8,9}$, Maho Haseda $^{9}$, Naoki Kondo ${ }^{9}$, Katsunori Kondo ${ }^{10,11}$, for the Japan Gerontological Evaluation Study group

\section{Affiliations}

${ }^{1}$ Osaka City University Graduate School of Human Life Science, Osaka, Japan

${ }^{2}$ Department of Public Health, Faculty and Graduate School of Medicine, Hokkaido University, Hokkaido, Japan

${ }^{3}$ School of Medicine, Hokkaido University, Hokkaido, Japan

${ }^{4}$ Clinical Research and Medical Innovation Center, Hokkaido University Hospital, Hokkaido, Japan

${ }^{5}$ Osaka City University School of Human Life Science, Osaka, Japan

${ }^{6}$ Department of Global Health Promotion, Tokyo Medical and Dental University, Tokyo, Japan

${ }^{7}$ Department of International Health and Collaboration, National Institute of Public Health, Saitama, Japan

${ }^{8}$ Behavioral Science Division, Center for Public Health Sciences, National Cancer Center, Tokyo, Japan

${ }^{9}$ Department of Health Education and Health Sociology, School of Public Health, The University of Tokyo, Tokyo, Japan

${ }^{10}$ Department of Social Preventive Medical Sciences, Center for Preventive Medical Sciences, Chiba University, Chiba, Japan 
${ }^{11}$ Department of Gerontological Evaluation, Center for Gerontology and Social Science, National Center for Geriatrics and Gerontology, Aichi, Japan

Corresponding author: Shigekazu Ukawa, Osaka City University Graduate School of

Human Life Science, 1-1-138 Sugimoto, Sumiyoshi-ku, Osaka, Japan. Telephone/Fax: +81

66605 2897, Email: ukawa@life.osaka-cu.ac.jp

Running title: Social patterns \& functional disability

\section{Word counts}

Abstract: 250 words

Manuscript: 2,993 words

Ethical approval/informed consent: The study protocol was approved by the Ethics Committee for Research on Human Subjects at Nihon Fukushi University, Japan (no. 1005), and the Hokkaido University Graduate School of Medicine Institutional Ethical Board for Epidemiological Studies (no. 14-024). 


\section{ABSTRACT}

Aim: To examine whether patterns of social participation vary in their associations with functional disability.

Methods: Data from 44,978 participants (22,750 men and 22,228 women) who participated in the 2010 Japan Gerontological Evaluation Study — a study of those aged $\geq 65$ years from 23 municipalities in eight prefectures - were analyzed. Social participation information was obtained at baseline with an 8-item questionnaire. Incidence of functional disability from 2010-2013 was defined as a new certification of eligibility for municipal public long-term care insurance. Social participation patterns were analyzed using exploratory factor analysis and participants were classified into quartiles of factor scores of social participation patterns. A competing risk model was used to calculate the hazard ratios and $95 \%$ confidence intervals for the incidence of functional disability in three years of follow-up. Results: Two social patterns were identified: sports groups/clubs and hobby groups and political groups/organizations and industry/trade associations. For both patterns, compared to participants in the lowest quartile, participants in the highest quartile were more likely to be male, college educated, high-income, and current drinkers. Both patterns were associated with reduced incidence of functional disability (adjusted hazard ratios for top quartile of sports and hobby pattern: $0.66,95 \%$ confidence interval: $0.59,0.74$; for political and industry/trade pattern: $0.81,95 \%$ confidence interval: $0.72,0.90 ; p$ for trend $<.001$ for both).

Conclusions: Those whose social participation patterns were characterized by frequent participation in sports groups/clubs and hobby groups or political groups/organizations and industry/trade associations were less likely to develop a functional disability. 
Keywords: aged, epidemiology, Japan, long-term care, social participation 


\section{Introduction}

Japan is one of the fastest-aging societies worldwide, and preventing the need for long-term care (LTC) is a pressing social issue. Persons aged older than 65 years are certified as needing LTC when they are independent in performing the basic activities of daily living but require some assistance in performing instrumental activities of daily living; the primary causes of certification are disease burdens such as stroke, and dementia, and frailty. ${ }^{1}$ The prevalence of people aged 65 years or older that were certified as needing LTC was $18.8 \%$ in 2017, which has been increasing. ${ }^{2}$ Since LTC insurance (LTCI) is a mandatory national insurance system in Japan, the issue has both societal and fiscal implications.

National and local governments have been promoting social participation by senior individuals to prevent the need for LTC. Social participation in multiple activities ${ }^{3,4}$ specifically, participation in local community, hobby, or sports organizations 4,5 —is protective against functional disability. More frequent participation in social activities was associated with a lower risk of incident disability ${ }^{3}$ in studies in Chicago, USA. An intervention to provide community salons was associated with lower incidence of functional decline in Aichi Prefecture in Japan. ${ }^{6}$

The details of social participation have not been as widely studied: holding a leadership role in an organization is associated with a reduced risk of dementia ${ }^{7}$ and functional disability; ${ }^{5}$ however, even fairly minimal participation (inactive or less than once a month) in some groups is associated with the maintenance of functional competence. ${ }^{8}$ Social participation activities conducted with others, such as neighbors or friends, was found to have a stronger protective effect than other leisure activities against the onset of noncommunicable disease among middle-aged Japanese adults. ${ }^{9}$ One study that examined 
patterns of activity among older Italian adults found that leisure could be organized into "surrounding" (e.g., socializing, attending church), "organized" (formal activities), and "indoor" (individual and home activities) style, ${ }^{10}$ with the best health observed among those participating in "organized style" activities.

The approach of considering activities individually or by frequency of participation has been valuable; however, it also has several conceptual limitations. It does not consider the variety of social participation possibilities and their interactions because people do not take part in isolated social activities. One method of identifying and examining broader social participation patterns is exploratory factor analysis - a statistical tool for aggregating interrelated variables into composite factors. These factors represent social participation patterns in the study population and help to distinguish individuals per the combination of social activities they choose to participate in. Social participation patterns may go further than individual activity exposures toward explaining the incidence of functional disability. Thus, our aim was to elucidate the association between social participation pattern scores and the further onset of LTC need via a large-scale nationwide cohort study in Japan.

\section{Methods}

Study population

Data from the 2010 Japan Gerontological Evaluation Study (JAGES) and incident functional disability within three years were used for this analysis. The study has been described in detail elsewhere. ${ }^{5}$ Briefly, 81,971 non-disabled people $(37,842$ men and 44,129 women) aged $\geq 65$ years from 23 municipalities, including urban, suburban, and rural areas in 8 (out of 47) prefectures throughout Japan were enrolled. The baseline survey was conducted between August 2010 and January 2012. The data were obtained through a self- 
administered questionnaire distributed and collected by mail (response rate $=66 \%$ ). Of the original cohort members, 36,993 participants with missing data for social participation were excluded; therefore, the data from 44,978 participants (22,750 men and 22,228 women) were analyzed in this study.

Data collection

Information on social participation and other lifestyle factors was obtained in the baseline questionnaire. Social participation involved eight items that are described in detail elsewhere: ${ }^{4}$ sports groups or clubs, hobby groups, volunteer groups, citizen/consumer groups, religious organizations, political groups/organizations, local community activities (including neighborhood associations, senior citizen clubs, or firefighting teams), and industry or trade associations. Participants were asked about their social participation: "Do you belong to any of the following groups or organizations?" If they said yes, they were asked how often they participated: every day, 2-3 times/week, 1 time/week, 1-2 times/month, a few times a year, or none. We converted all answers into times per week $(7,2.5,1,0.35,0.05$, or 0 times/week).

Functional non-disability was defined as not a certificate of needing LTCI benefit along with our previous studies. ${ }^{4-6,13}$ All individuals aged $\geq 65$ years are eligible for the insurance program regardless of their income status. ${ }^{14}$ The data on the incidence of functional disability from 2010 to 2013 were obtained from municipal public LTCI system databases, with those who were newly certified as eligible for the LTCI benefit considered as functionally disabled. The certification of eligibility for the LTCI benefit is judged based on a nationally standardized procedure including a physician's examination and evaluation of physical and cognitive functions. ${ }^{14}$

Statistical analysis 
Social participation patterns based on the eight social participation items were analyzed using exploratory factor analysis with the SAS PROC FACTOR procedure, applying the principal factors method with two factors and a promax (oblique) rotation. We treated the frequency data as continuous. For determination of the number of factors to be retained, we first conducted a principal factor analysis, and then considered components with an eigenvalue $>1.0$ as well as the scree test results and interpretability of the factors. The factor scores - a linear composite of the optimally weighted variables under analysis — were also calculated for each participant within the same SAS procedure and standardized to a mean value of 0.0 and a standard deviation of 1.0. Each participant was assigned a factor score for each identified pattern. Participants were then classified into quartiles of factor scores of social participation patterns. Bivariate analyses were conducted, examining the demographic and other predictors of social participation patterns using chi-square tests.

A competing risk model was used to calculate the hazard ratios (HRs) and $95 \%$ confidence intervals (CIs) for incidence of functional disability. ${ }^{15}$ We treated death as a competing event because all-cause mortality and functional disability share a lack of social involvement as a common risk factor. We used the SAS PROC PHREG procedure to calculate HRs, incorporating Fine and Gray's model using the eventcode option. Trend $p$-values were calculated to assess associations between the four categories of social participation patterns and the incidence of functional disability. Multiplicative interaction terms between sex and each social participation pattern were evaluated in the fully adjusted models using likelihood ratio tests.

The following variables measured at baseline were included in the model ${ }^{4,5}$ : municipality; sex (man or woman); age (65-69, 70-74, 75-79, or $\geq 80$ years); marital status 
(married, widowed/divorced, single, or other/missing); employment status (working, not working, or missing); educational attainment $(<6,6-9,10-12, \geq 13$ years, or other/missing); equivalized incomes $(<2.00,2.00-3.99, \geq 4.00$ million yen, or missing); smoking status (current, former, never, or missing); drinking status (current, former, never, or missing); depressive tendencies (presence, absence, or missing); functional capacity (high, low, or missing); subjective cognitive complaint (presence, absence, or missing); social support (presence or absence/missing); and medical history of cancer (yes, no/missing), heart disease (presence or absence/missing), stroke (yes or no/missing), respiratory disease (presence or absence/missing), arthritic disorder (presence or absence/missing), and trauma and/or bone fracture (presence or absence/missing).

Annual equivalized household income was adjusted for household size by dividing the income by the square root of the number of people in that household. We assessed depressive symptoms with the Japanese short version of the Geriatric Depression Scale (GDS-15) — a self-rating depression-screening tool for older people that has been validated among Japanese older people. ${ }^{11}$ A total score of six or higher was treated as indicating depressive tendencies. Functional capacity was assessed by a validated questionnaire of the Tokyo Metropolitan Institute of Gerontology Index of Competence, ${ }^{12}$ and total scores ranged from 0 to 13 , with a score of $\geq 10$ considered as high competence. Presence of emotional and/or institutional social support was measured by the following two questions: "Do you have someone who listens to your concerns and complaints?" and "Do you have someone who looks after you when you are sick and have to stay in bed for a few days?" If participants received social support from someone, it denoted the presence of social support. Subjective cognitive complaints were assessed with following three questions: "Do you often get into 
trouble when you leave your belongings behind somewhere?," "Do you often get times or places confused?," and "Do you often forget things that happened recently (e.g., what you had for breakfast)?" Presence of subjective cognitive complaints was defined by answering yes to at least one item.

An alpha level of .05 was considered significant. All analyses were performed using SAS 9.4 (SAS Institute Inc., Cary, NC, USA).

Ethical considerations

The study protocol for the JAGES project was approved by the Ethics Committee for Research on Human Subjects at Nihon Fukushi University, Japan (no. 10-05), and the Hokkaido University Graduate School of Medicine Institutional Ethical Board for Epidemiological Studies (no. 14-024).

\section{Results}

Two social participation patterns were extracted using an exploratory factor analysis. The factor-loading matrix for social participation patterns and social participation items is shown in Table 1. The first pattern had a higher loading of sports groups or clubs and hobby groups. The second pattern had a higher loading of political groups/organizations and industry/trade associations. These two patterns were labeled as follows: The sports and hobby pattern and the political and industry pattern.

Participants' baseline characteristics are shown per the factor score quartiles of the two social participation patterns in Tables 2 and 3. For both the sports groups or clubs and hobby and the political groups/organizations and industry/trade associations patterns, compared to participants in the lowest quartile, participants in the highest quartile were older; had higher baseline functional capacity; and were more likely to be male, working, college 
educated, high-income, and current drinkers; had more comorbidities; and were less likely to have cognitive complaints $(p<.001)$. For the sports groups or clubs and hobby pattern, participants in the highest quartile were less likely to be current smokers $(p<.001)$.

The median follow-up time was 2.9 years. During a total of 124,870 person-years, 3,221 participants experienced incident functional disability. Table 4 shows the HR and 95\% CI of functional disability of the two social participation patterns by score quartile among Japanese older participants. After adjusting for potential confounders such as age; sex; municipality; marital status; employment status; educational attainment; annual equivalized household income; smoking status; drinking status; depressive tendencies; functional capacity; social support; subjective cognitive complaints; and medical history of cancer, heart disease, stroke, respiratory disease, arthritic disorder, and trauma and/or bone fracture, the HRs for the sports groups or clubs and hobby pattern were 0.88 (95\% CI: $0.80-0.98$ ) for the second, 0.84 (95\% CI: 0.76-0.93) for the third, and 0.66 (95\% CI: 0.59-0.74) for the fourth quartile compared with the bottom quartile ( $p$ for trend $<.001)$. The political groups/organizations and industry/trade associations for the second (HR: 0.89, 95\% CI: 0.800.99), third (HR: $0.85,95 \%$ CI: $0.77-0.94$ ), and fourth (HR: $0.81,95 \%$ CI: $0.72-0.90$ ) quartile also showed a decreased risk for the incidence of functional disability ( $p$ for trend $<.001)$. No significant interactions between sex and social participation patterns were found ( $p$ for interaction $=.44$ for sports groups or clubs and hobby pattern; 0.99 for political groups/organizations and industry/trade associations pattern).

\section{Discussion}

We derived two major social participation patterns-“sports groups or clubs and hobby groups" and "political groups/organizations and industry/trade associations"-from a 
large-scale nationwide cohort study in Japan. These two social participation patterns were inversely associated with incident functional disability. Even when we set our outcome to a severe disability defined as care levels $2-5^{17}$, the same trends were found in these two social participation patterns (data not shown). Only a few previous studies have addressed broader patterns of social participation. One study of a Finnish-Swedish population found four patterns: voluntary associational activity, friendship, religious association, and hobby participation; ${ }^{16}$ while, in our analysis only two patterns were identified. A study of leisure patterns in Italy produced the broader categories of "surrounding," "organized," and "indoor" style; ${ }^{10}$ consistent with our analysis, sports and other activities were included in the “organized style," which was associated with the best health.

Overall, the results are largely consistent with previous studies of individual types of social participation. For the sports groups or clubs and hobby groups pattern, our results were consistent with our former prospective cohort study-2003 AGES, in Japan ${ }^{4,5}$ — which reported an inverse association between incident functional disability and participation in sports and hobby organizations. Those who participated in a sports club (adjusted HR: 0.66; 95\% CI: 0.51-0.85) or hobby group (adjusted HR: 0.69; 95\% CI: 0.55-0.87) were less likely to be functionally disabled than other participants in the same cohort. ${ }^{5}$ The benefit of participation in a sports club and hobby group in protecting against functional disability may be owing to not only the physical ${ }^{17}$ or recreational activities performed, ${ }^{18}$ but also the social benefits obtained, such as instrumental or emotional support. ${ }^{4}$ Previous studies have found participation in alumni groups, volunteer groups, neighborhood community associations, hobby clubs, and cultural clubs to be associated with better functional competence. ${ }^{8,19}$ In a Korean study, participation in senior citizen clubs or centers was associated with reduced 
cognitive decline. ${ }^{20}$ One study found stronger effects in women than men; ${ }^{19}$ however, we did not observe any sex-related effects.

In contrast to the results of our previous prospective cohort study ${ }^{5}$, which indicated that there were no significant associations between participation in political organizations or groups (adjusted HR: 1.24; 95\% CI: 0.97-1.58) and industrial or trade associations (adjusted HR: 1.13 ; 95\% CI: $0.88-0.1 .44)$ and incident disability, we found that higher political groups/organizations and industry/trade associations pattern scores were associated with significantly decreased incident functional disability. These inconsistent results may be owing to an interaction or the synergistic effect of multiple social participations. Although the potential pathways are still unclear, better social relationships, obtaining formal/informal social support, and social ties may be linked to positive health behaviors. ${ }^{21}$ However, further studies are needed to elucidate the direct mechanisms of effect. A Chinese study found that playing cards/mahjongg and organized social activities were protective against functional decline. ${ }^{22}$ Both types of activity were mediated by cognitive ability; however, the first was partially mediated by positive emotions and the second by physical exercise. ${ }^{22}$ Analysis of the Ohsaki Cohort of 2006 found that cognitive activity, walking, psychological state, and social support all mediated the effect of volunteering, hobbies, and neighborhood associations on functional disability. ${ }^{23}$ In addition, political groups/organizations and industry/trade associations serve to link the social capital that individuals build in their relationships with institutions and individuals that have relative power over them. ${ }^{24}$ Although our findings are not directly comparable, one report from Denmark ${ }^{25}$ and two reports from Sweden ${ }^{26,27}$ found an association between neighborhood social capital-defined by neighborhood voting participation rates - and lower all-cause mortality as well as mortality 
due to coronary heart disease, psychiatric disorders, cancer, stroke, chronic lower respiratory diseases, and type II diabetes. ${ }^{27}$ A Japanese study also found that higher community mistrust was associated with the onset of disability, ${ }^{13}$ and this association was possibly mediated by social participation.

The strengths of this study include its prospective cohort design and the inclusion of participants from throughout Japan, as well as a very complete follow-up for functional disability. However, the current study contains some limitations that warrant consideration. First, our previous report showed a lack of social participation to be a risk factor for all-cause mortality. ${ }^{28}$ Therefore, as all-cause mortality and functional disability share a lack of social involvement as a common risk factor, this may lead to an underestimation of its effect on incident functional disability. To reduce this bias, we conducted a competing risk analysis. Second, the information on social participation was collected only at baseline and was not updated during the study period. National and local governments have undertaken efforts to promote social participation for older individuals in Japan $;^{29}$ thus, some individuals who became more socially active might have been misclassified; if non-differential, this may have diminished the estimated HR. Third, although the participants were exclusively independent older individuals, we cannot rule out the possibility of reverse causality owing to the short follow-up period of three years. Even with control for functional status at baseline, residual confounding may be produced as those who are healthier are more likely to participate in social activities. Most likely, however, this concern would not differentially affect one social participation pattern or another. Fourth, cognitive function could reduce the response rate and affect the quality of responses, which might lead to those with poorer functional prognosis and less social participation to be underrepresented in the study and, among those 
participating, having differentially poorer quality of data. Fifth, although we used the KaiserGuttman criteria, deciding the number of factors was subjective and ambiguous. However, even if we set three or four factors in the principal factor analysis, the first two patterns were similar. Therefore, the two social participation patterns we extracted might have robustness. Finally, since social participation patterns were extracted from data obtained within the study population, it is reasonable that the results are not reproduced in different populations. Despite this drawback, an advantage of this approach is that the description of social participation patterns allowed for the promotion of changes to be focused on the participation of social activities that are readily recognized by the target group.

In conclusion, this nationwide cohort study indicates that those whose social participation patterns were characterized by frequent participation in sports groups or clubs and hobby groups, or political groups/organizations and industry/trade associations, were less likely to develop a functional disability. Generally speaking, the results have been consistent across different settings and countries $;^{30}$ however, further research is needed to determine whether these social patterns are specific to Japan or are more broadly applicable. Grouping activities into those with similar effects can help in designing optimal interventions to improve participation and health; for instance, a "one from column A, one from column B" approach may be best for maintaining health. Consequently, the present findings can be used to assess types of social participation and methods for preventing the need for LTC. 
Acknowledgments: We thank the members of the JAGES project. Editorial support, in the form of medical writing; assembling tables; creating high-resolution images based on authors' detailed directions; collating author comments; copyediting; fact checking; and referencing was provided by Editage, Cactus Communications.

Funding: This study used data from the JAGES, which was supported by the Japan Society for the Promotion of Science; KAKENHI (grant nos. JP15H01972, JP 18H05389, JP17K19778, JP17H04129, and JP18H05389), a Health Labour Sciences Research grant (no. H28-Choju-Ippan-002), the Japan Agency for Medical Research and Development (nos. JP17dk0110017, JP18dk0110027, JP181s0110002, and JP18le0110009), and Research Funding for Longevity Sciences from the National Center for Geriatrics and Gerontology (no. 29-42).

Disclosure statement: The authors declare no conflict of interest. 


\section{References}

1 Cabinet Office Government of Japan. Annual Report on the Aging Society: 2017. Tokyo, Japan. [Cited 6 Oct 2017]. Available from: http://www8.cao.go.jp/kourei/whitepaper/w2017/zenbun/29pdf_index.html.

2 Ministry of Health, Labour and Welfare. Survey of Long-term Care Benefit Expenditures: 2017. Toyo, Japan. [Cited 1 Aug 2019]. Available from: https://www.mhlw.go.jp/toukei/list/45-1b.html.

3 James BD, Boyle PA, Buchman AS, Bennett DA. Relation of late-life social activity with incident disability among community-dwelling older adults. J Gerontol A Biol Sci Med Sci 2011; 66: 467-473.

4 Kanamori S, Kai Y, Aida J, et al. Social participation and the prevention of functional disability in older Japanese: the JAGES cohort study. PLoS One 2014; 9: e99638.

5 Ashida T, Kondo N, Kondo K. Social participation and the onset of functional disability by socioeconomic status and activity type: The JAGES cohort study. Prev Med 2016; 89: 121128.

6 Hikichi H, Kondo N, Kondo K, Aida J, Takeda T, Kawachi I. Effect of a community intervention programme promoting social interactions on functional disability prevention for older adults: propensity score matching and instrumental variable analyses, JAGES Taketoyo study. J Epidemiol Community Health 2015; 69: 905-910.

7 Nemoto Y, Saito T, Kanamori S, et al. An additive effect of leading role in the organization between social participation and dementia onset among Japanese older adults: the AGES cohort study. BMC Geriatr 2017; 17: 297. 
8 Nonaka K, Suzuki H, Murayama H, et al. For how many days and what types of group activities should older Japanese adults be involved in to maintain health? A 4-year longitudinal study. PLoS One 2017; 12: e0183829.

9 Oshio T, Kan M. Preventive impact of social participation on the onset of noncommunicable diseases among middle-aged adults: a 10-wave hazards-model analysis in Japan. Prev Med 2019; 118: 272-278.

10 Gagliardi C, Spazzafumo L, Papa R, Marcellini F. Changes in leisure styles and satisfaction of older people: a five years follow-up. Int J Aging Hum Dev 2012; 75: 185-215.

11 Wada T, Ishine M, Kita T, Fujisawa M, Matsubayashi K. Depression screening of elderly community-dwelling Japanese. J Am Geriatr Soc 2003; 51: 1328-1329.

12 Koyano W, Shibata H, Nakazato K, Haga H, Suyama Y. Measurement of competence: reliability and validity of the TMIG Index of Competence. Arch Gerontol Geriatr 1991; 13: $103-116$.

13 Aida J, Kondo K, Kawachi I, et al. Does social capital affect the incidence of functional disability in older Japanese? A prospective population-based cohort study. J Epidemiol Community Health 2013; 67: 42-47.

14 Tsutsui T, Muramatsu N. Japan's universal long-term care system reform of 2005: containing costs and realizing a vision. J Am Geriatr Soc 2007; 55: 1458-1463.

15 Fine JP, Gray RJ. A proportional hazards model for the subdistribution of a competing risk. J Am Stat Assoc 1999; 94: 496-509.

16 Hyyppä MT, Mäki J. Social participation and health in a community rich in stock of social capital. Health Educ Res 2003; 18: 770-779. 
17 Matsunaga T, Naito M, Wakai K, et al. Leisure-time physical activity and risk of disability incidence: a 12-year prospective cohort study among young elderly of the same age at baseline. J Epidemiol 2017; 27: 538-545.

18 Takeda T, Kondo K, Hirai H. [Psychosocial risk factors involved in progressive dementiaassociated senility among the elderly residing at home. AGES project: three year cohort longitudinal study]. Nihon Koshu Eisei Zasshi 2010; 57: 1054-1065.

19 Tomioka K, Kurumatani N, Saeki K. The differential effects of type and frequency of social participation on IADL declines of older people. PLoS One 2018; 13: e0207426.

20 Lee SH, Kim YB. Which type of social activities may reduce cognitive decline in the elderly? A longitudinal population-based study. BMC Geriatr 2016; 16: 165.

21 Umberson D, Crosnoe R, Reczek C. Social relationships and health behavior across life course. Annu Rev Sociol 2010; 36: 139-157.

22 Gao M, Sa Z, Li Y, et al. Does social participation reduce the risk of functional disability among older adults in China? A survival analysis using the 2005-2011 waves of the CLHLS data. BMC Geriatr 2018; 18: 224.

23 Otsuka T, Tomata Y, Zhang S, et al. Association between social participation and incident risk of functional disability in elderly Japanese: the Ohsaki Cohort 2006. J Psychosom Res 2018; 111: 36-41.

24 Szreter S, Woolcock M. Health by association? Social capital, social theory, and the political economy of public health. Int J Epidemiol 2004; 33: 650-667.

25 Poulsen T, Siersma VD, Lund R, Christensen U, Vass M, Avlund K. Impact of social capital on 8-year mortality among older people in 34 Danish municipalities. J Aging Health 2012; 24: 1203-1222. 
26 Sundquist J, Hamano T, Li X, Kawakami N, Shiwaku K, Sundquist K. Neighborhood linking social capital as a predictor of psychiatric medication prescription in the elderly: a Swedish national cohort study. J Psychiatr Res 2014; 55: 44-51.

27 Sundquist K, Hamano T, Li X, Kawakami N, Shiwaku K, Sundquist J. Linking social capital and mortality in the elderly: a Swedish national cohort study. Exp Gerontol 2014; 55: 29-36.

28 Ishikawa Y, Kondo N, Kondo K, Saito T, Hayashi H, Kawachi I. Social participation and mortality: does social position in civic groups matter? BMC Public Health 2016; 16: 394.

29 Ministry of Health, Labour and Welfare. Community-based Integrated Care Systems: 2017. Tokyo, Japan. [Cited 17 Oct 2017]. Available from: http://www.mhlw.go.jp/stf/seisakunitsuite/bunya/hukushi_kaigo/kaigo_koureisha/chiikihoukatsu/.

30 Willie-Tyndale D, Holder-Nevins D, Mitchell-Fearon K, et al. Participation in social activities and the association with socio-demographic and health-related factors among community-dwelling older adults in Jamaica. J Cross Cult Gerontol 2016; 31: 427-447. 
Table 1 Factor loading matrix for the two major social participation patterns, JAGE Study

\begin{tabular}{lcc}
\hline & $\begin{array}{c}\text { sports groups or clubs } \\
\text { and hobby groups }\end{array}$ & $\begin{array}{c}\text { political } \\
\text { groups/organizations } \\
\text { and industry/trade } \\
\text { associations }\end{array}$ \\
& & \\
\hline Political groups/organization & 0.03 & $\mathbf{0 . 5 4}$ \\
Industry or trade associations & -0.01 & $\mathbf{0 . 4 8}$ \\
Volunteer groups & 0.11 & 0.28 \\
Local community activities & & 0.16 \\
Religious organizations & 0.31 & 0.22 \\
Sports groups or clubs & -0.04 & -0.06 \\
Citizen/consumer groups & & 0.24 \\
\hline
\end{tabular}

Factors with loadings $\geq 0.4$ are in boldface. 
Table 4 Hazard ratios and 95\% confidence intervals of functional disability of two social participation patterns by score quartile among Japanese elderly participants

$\begin{array}{lllll}\text { Quartile 1 } & \text { Quartile 2 } & \text { Quartile 3 } & \text { Quartile 4 } & P \text { for trend }\end{array}$

\begin{tabular}{|c|c|c|c|c|c|}
\hline \multicolumn{6}{|c|}{ Sports groups or clubs and hobby groups } \\
\hline Person-years & 39,090 & 21,330 & 32,170 & 32,280 & \\
\hline Number of cases & 1688 & 497 & 600 & 436 & \\
\hline Number of deaths & 638 & 196 & 196 & 128 & \\
\hline $\mathrm{HR}(95 \% \mathrm{CI})^{1}$ & 1.00 & $0.67(0.60,0.74)^{*}$ & $0.57(0.52,0.62)^{*}$ & $0.42(0.38,0.47)^{*}$ & $<.001$ \\
\hline $\mathrm{HR}(95 \% \mathrm{CI})^{2}$ & 1.00 & $0.88(0.80,0.98)^{*}$ & $0.84(0.76,0.93)^{*}$ & $0.66(0.59,0.74)^{*}$ & $<.001$ \\
\hline
\end{tabular}

Political groups/organizations and industry/trade associations pattern

$\begin{array}{llllll}\text { Person-years } & 40,568 & 20,693 & 31,566 & 32,042 & \\ \text { Number of cases } & 1611 & 445 & 638 & 527 & \\ \text { Number of deaths } & 627 & 168 & 206 & 157 & \\ \text { HR }(95 \% \mathrm{CI})^{1} & 1.00 & 0.70(0.61,0.76)^{*} & 0.61(0.56,0.67)^{*} & 0.54(0.49,0.60)^{*} & <.001 \\ \text { HR }(95 \% \mathrm{CI})^{2} & 1.00 & 0.89(0.80,0.99)^{*} & 0.85(0.77,0.94)^{*} & 0.81(0.72,0.90)^{*} & <.001\end{array}$

HR hazard ratio, CI confidence interval. $* \mathrm{P}<.05$.

${ }^{1}$ competing risk model adjusted for age, sex, and municipality.

${ }^{2}$ competing risk model adjusted for age, sex, municipality, marital status, employment status, educational attainment, annual equivalized household income, smoking status, drinking status, depressive tendency, 
functional capacity, social support, subjective cognitive complaints, and medical history of cancer, heart disease, stroke, respiratory disease, arthritic disorder, and trauma and/or bone fracture. 
Table 3 Factors associated with political groups/organizations and industry/trade associations patterns at baseline by score quartiles

\section{Quartile 1 Quartile 2 Quartile 3 Quartile 4}

Variable $\quad n=15,107 \quad n=7,398 \quad n=11,233 \quad n=11,240 \quad p$-value

Sex

Men

53.3

50.5

50.0

43.1

$<.001$

Age (years)

65-69

34.2

41.1

38.3

38.8

$<.001$

70-74

20.9

24.4

24.2

25.9

75-79

15.9

14.1

16.0

16.8

$\geq 80$

29.0

20.4

21.6

18.5

Marital status

Married

67.7

73.3

74.7

76.2

$<.001$

Widowed/divorced

25.8

21.9

20.9

19.7

Single

2.0

1.6

1.4

1.0

Other/missing

4.5

3.2

3.0

3.1

\section{Employment status}

Working

Not working
17.7

69.5

69.7

66.5

65.3 

Missing
12.9
9.0
8.1
8.0

Educational attainment (years)

$\begin{array}{lccccc}<6 & 4.0 & 2.1 & 1.3 & 1.4 & <.001 \\ 6-9 & 51.9 & 44.5 & 42.7 & 37.5 \\ 10-12 & 27.6 & 34.0 & 35.7 & 37.1 \\ \geq 13 & 12.2 & 16.2 & 17.3 & 20.7 \\ \text { Other/missing } & 4.4 & 3.1 & 3.0 & 3.3\end{array}$

Equivalized incomes (million yen)

$\begin{array}{lccccc}<2.00 & 43.2 & 42.3 & 37.8 & 37.1 & <.001 \\ 2.00-3.99 & 29.4 & 35.2 & 37.9 & 38.5 & \\ \geq 4.00 & 60.0 & 6.6 & 8.0 & 8.6 & \\ \text { Missing } & 21.4 & 15.9 & 16.2 & 15.8\end{array}$

Smoking status

\begin{tabular}{|c|c|c|c|c|}
\hline Current & 11.7 & 11.7 & 10.4 & 11.2 \\
\hline Former & 25.8 & 28.2 & 27.9 & 29.7 \\
\hline Never & 53.3 & 53.4 & 54.9 & 52.7 \\
\hline Missing & 9.3 & 7.0 & 6.8 & 6.5 \\
\hline
\end{tabular}

Drinking status

Current

27.6

33.8

36.5

42.4

$<.001$ 


$\begin{array}{lcccc}\text { Former } & 4.0 & 3.6 & 3.2 & 2.9 \\ \text { Never } & 63.3 & 58.1 & 56.0 & 4.3 \\ \text { Missing } & 5.1 & 4.5 & 4.3 & 4.5\end{array}$

GDS-15

$\geq 6$

$<6$

Missing

TMIG

$\geq 10$

$<10$

Missing

Subjective cognitive

complaints

Presence

Absence

Missing

Social support

Presence

Medical history of
24.9

60.1

15.0

12.7

16.4

71.0

73.5

14.1

10.7

$<.001$

(1)

15.0

63.5

80.2

84.4

88.0

$<.001$

25.0

11.3

7.8

5.0

11.5

8.5

7.8

7.1 
Presence

Heart disease

Presence

Stroke

Presence

Respiratory disease

Presence

Arthritic disorder

Presence

Trauma and/or bone

fracture

Presence
4.9

4.7

13.3

11.9

11.7

10.8

$<.001$

$\begin{array}{ll}4.1 & 3.6\end{array}$

$<.001$

( 
Table 4 Hazard ratios and 95\% confidence intervals of functional disability of two social participation patterns by score quartile among Japanese elderly participants

$\begin{array}{lllll}\text { Quartile 1 } & \text { Quartile 2 } & \text { Quartile 3 } & \text { Quartile 4 } & P \text { for trend }\end{array}$

\begin{tabular}{|c|c|c|c|c|c|}
\hline \multicolumn{6}{|c|}{ Sports groups or clubs and hobby groups } \\
\hline Person-years & 39,090 & 21,330 & 32,170 & 32,280 & \\
\hline Number of cases & 1688 & 497 & 600 & 436 & \\
\hline Number of deaths & 638 & 196 & 196 & 128 & \\
\hline $\mathrm{HR}(95 \% \mathrm{CI})^{1}$ & 1.00 & $0.67(0.60,0.74)^{*}$ & $0.57(0.52,0.62)^{*}$ & $0.42(0.38,0.47)^{*}$ & $<.001$ \\
\hline $\mathrm{HR}(95 \% \mathrm{CI})^{2}$ & 1.00 & $0.88(0.80,0.98)^{*}$ & $0.84(0.76,0.93)^{*}$ & $0.66(0.59,0.74)^{*}$ & $<.001$ \\
\hline
\end{tabular}

Political groups/organizations and industry/trade associations pattern

$\begin{array}{llllll}\text { Person-years } & 40,568 & 20,693 & 31,566 & 32,042 & \\ \text { Number of cases } & 1611 & 445 & 638 & 527 & \\ \text { Number of deaths } & 627 & 168 & 206 & 157 & \\ \text { HR }(95 \% \mathrm{CI})^{1} & 1.00 & 0.70(0.61,0.76)^{*} & 0.61(0.56,0.67)^{*} & 0.54(0.49,0.60)^{*} & <.001 \\ \text { HR }(95 \% \mathrm{CI})^{2} & 1.00 & 0.89(0.80,0.99)^{*} & 0.85(0.77,0.94)^{*} & 0.81(0.72,0.90)^{*} & <.001\end{array}$

HR hazard ratio, CI confidence interval. $* \mathrm{P}<.05$.

${ }^{1}$ competing risk model adjusted for age, sex, and municipality.

${ }^{2}$ competing risk model adjusted for age, sex, municipality, marital status, employment status, educational attainment, annual equivalized household income, smoking status, drinking status, depressive tendency, 
functional capacity, social support, subjective cognitive complaints, and medical history of cancer, heart disease, stroke, respiratory disease, arthritic disorder, and trauma and/or bone fracture. 